CrossMark < click for updates

Cite this: Phys. Chem. Chem. Phys., $2016,18,26495$

Received 5th August 2016, Accepted 26th August 2016

DOI: $10.1039 / \mathrm{c} 6 \mathrm{cp} 05453 \mathrm{f}$

www.rsc.org/pccp

\title{
Attachment of composite porous supra-particles to air-water and oil-water interfaces: theory and experiment
}

\author{
Vesselin N. Paunov, * Hamza Al-Shehri and Tommy S. Horozov
}

\begin{abstract}
We developed and tested a theoretical model for the attachment of fluid-infused porous supra-particles to a fluid-liquid interface. We considered the wetting behaviour of agglomerated clusters of particles, typical of powdered materials dispersed in a liquid, as well as of the adsorption of liquid-infused colloidosomes at the liquid-fluid interface. The free energy of attachment of a composite spherical porous supra-particle made from much smaller aggregated spherical particles to the oil-water interface was calculated. Two cases were considered: (i) a water-filled porous supra-particle adsorbed at the oil-water interface from the water phase, and, (ii) an oil-filled porous supra-particle adsorbed at the oil-water interface from the oil-phase. We derived equations relating the three-phase contact angle of the smaller "building block" particles and the contact angle of the liquid-infused porous supra-particles. The theory predicts that the porous supra-particle contact angle attached at the liquid interface strongly depends on the type of fluid infused in the particle pores and the fluid phase from which it approaches the liquid interface. We tested the theory by using millimetre-sized porous supra-particles fabricated by evaporation of droplets of polystyrene latex suspension on a pre-heated super-hydrophobic surface, followed by thermal annealing at the glass transition temperature. Such porous particles were initially infused with water or oil and approached to the oil-water interface from the infusing phase. The experiment showed that when attaching at the hexadecane-water interface, the porous supra-particles behaved as hydrophilic when they were pre-filled with water and hydrophobic when they were pre-filled with hexadecane. The results agree with the theoretically predicted contact angles for the porous composite supra-particles based on the values of the contact angles of their building block latex particles measured with the Gel Trapping Technique. The experimental data for the attachment of porous supra particles to the air-water interface from both air and water also agree with the theoretical model. This study gives important insights about how porous particles and particle aggregates attach to the oil-water interface in Pickering emulsions and the air-water surface in particle-stabilised aqueous foams relevant in ore flotation and a range of cosmetic, pharmaceutical, food, home and personal care formulations.
\end{abstract}

\section{Introduction}

The wettability of the powder particles by liquids has been a subject of strong interest during the last few decades due to its importance in formulating cosmetic, pharmaceutical and food products, paints and building materials, waste water treatment, secondary oil recovery as well as flotation of ores. ${ }^{1-4}$ Ramsden $^{5}$ and Pickering ${ }^{6}$ reported the first emulsion systems stabilised by solid colloid particles. When colloidal particles attach to liquid surfaces, they decrease the free energy of the system. ${ }^{7,8}$ The adsorption energy of a colloid particle at the liquid interface (e.g. air-water or oil-water), $\Delta F$, could be many orders of

Department of Chemistry, University of Hull, Hull, HU67RX, UK.

E-mail:v.n.paunov@hull.ac.uk; Fax: +44 (0)1482466410; Tel: +44 (0)1482 465660 magnitude higher than the thermal energy, $k T$. This indicates that colloid particles can attach irreversibly to the liquid-fluid interface. For that reason, colloid particles are used as emulsion stabilisers $^{9-13}$ and foaming agents giving super-stable foams. ${ }^{14-16}$ The particle wettability, surface chemistry, and shape have influence on how strongly the particles attach at the interface and determine their efficiency as emulsifiers and foam-stabilisers.

Most of the theoretical considerations for the attachment of colloid particles at liquid surface have been done on solid spherical particles with smooth surfaces. ${ }^{7,17,18}$ Although, the adsorption of surface anisotropic Janus particles, ${ }^{19}$ and that of particles of non-spherical shape ${ }^{20}$ have also been considered, a detailed theoretical description of the adsorption of porous spherical particles has not been reported in the literature. Although some MD-based simulation approaches for studying 
the assembly of nanoparticles and Janus particles at liquid surfaces have already been explored, ${ }^{38-41}$ no general insights on the attachment of composite porous particles at liquid interfaces have been developed so far due to the complexity of their geometry.

In this study, we develop an analytical theoretical model of the attachment of porous supra-particles from water or oil to the oil-water interface. Composite porous particles made from smaller colloidal particles will be considered as a model for a particle aggregate, which is a common occurrence in powdered materials. Our aim is to derive an equation connecting the three-phase contact angle, $\theta$, of the supra-particle attached to the liquid interface and the three-phase contact angle, $\theta_{0}$ of its building blocks, i.e. the smaller colloid particles from which it is formed. Using reasonable assumptions, we derive simple analytical equations for the supra-particle attachment energy to the liquid interface which enable us to study the importance of the initial fluid phase, from which the supra-particle approaches the liquid interface. The effect of the particle radius and the contact angle of the building blocks of the supra-particle on its wettability is investigated and the energy of attachment of a single colloid particle is compared with the energy of attachment of a spherical aggregate of such particles. Furthermore, the effect of the surface packing density of small particles at the supra-particle surface, on its effective three-phase contact angle is investigated. Very similar considerations would apply to the attachment behaviour of spherical composite colloidosomes, whose effective contact angle and free energy of attachment at the liquid interface can be described with the same formulae as the composite supra-particles.

One of the main assumptions of this work is that the supraparticles attach to the liquid interface through a single layer of small colloid particles on the supra-particle surface. In this case, the liquid-fluid interface does not penetrate the core of the porous particles. This assumption seems reasonable as the potential penetration of the liquid surface front inside the porous particle would require the detachment of the surface layer of colloid particles, hence, a very high energy input, much larger than the thermal energy would be required for such penetration of the liquid surface front to occur inside the composite supra-particle. This means that a supra-particle (or a particle aggregate) is unlikely to spontaneously adjust the position of the liquid interface further than the surface layer of small colloid particles (building blocks).

We did several types of experiments to study the effect of the surface structure, porosity and the infusion of the porous particles by one of the liquid phases on their adsorption at the fluid-liquid interfaces. ${ }^{37}$ We prepared model porous supra particles by evaporating latex micro-particle suspensions on a super-hydrophobic surface followed by their partial fusion. ${ }^{26,27}$ The aim was to accumulate experimental data about the way of attachment of porous supra-particles to liquid surfaces and to explore the link between the wettability of the supra-particle building blocks (smaller colloid particles) and the macroscopic (apparent) contact angle of the porous particles at liquid surfaces.

The contact angles of the individual colloid particles at air-water and oil-water interfaces were investigated using the gel trapping technique (GTT) ${ }^{21-25}$ by injecting a small sample of the particle suspension in a spreading solvent near the liquid interface through the polar or non-polar phase. In a separate series of experiments, the porous supra-particle contact angles were also studied. Also the effect of the fluid phase by which the porous particles are initially infused on their attachment at the liquid-fluid interface was experimentally studied. Finally, the link between the wettability of the particle building blocks and the macroscopic (apparent) contact angle of the porous particles at liquid surfaces was investigated. The porous supra-particle wettability at both oil-water and air-water interfaces was investigated. For example, the supra-particle wettability at the oil-water interface was studied using porous supra-particles initially infused with water (from the aqueous phase) or infused with oil (from the oil phase). For the air-water interface, the attachment of porous supra-particles initially infused with water (from the aqueous phase) and dry porous supra-particles (coming from the air) to the air-water surface was investigated.

In the following section, the attachment free energy of a porous supra-particle (or a colloidosome of the same surface layer of smaller particles and fluid infusion) at the liquid interface will be discussed. The supra-particle macroscopic contact angles will be related to the contact angle of the individual building block particles. The impact of the surface packing arrangement (hexagonal, square or random close packing) of the supra-particle building blocks on the contact angle and the free energy of its attachment to the liquid-fluid interface will be studied. The importance of the initial fluid phase infused into these porous supra-particles and its effect on the particle free energy of attachment at the liquid interface will also be discussed.

\section{Theoretical background}

Here we analyse the process of the attachment of a porous spherical supra-particle to an oil-water interface. Two cases will be considered: (i) when the porous supra-particle is initially in the aqueous phase (Scheme $1 \mathrm{~A}$ and $\mathrm{B}$ ); and (ii) when the porous supra-particle is initially in the oil phase (Scheme $1 \mathrm{C}$ and D). In both cases, it is assumed that the porous supra-particle is infused with the same fluid from which the oil-water interface is approached, i.e. water in case (i) and oil in case (ii). The porous composite supra-particle will be assumed to be spherical (with radius $R$ ) and encased by a shell of a monolayer of closelypacked spherical particles (with radius $a$ ). Our aim here is to find the connection between the three-phase contact angle, $\theta_{0}$, of the colloid particles in the particle monolayer and the effective three-phase contact angle, $\theta$, of the supra-particle when attached to an oil-water interface. Hereafter, the contact angles $\theta$ and $\theta_{0}$ are defined through the water phase. All of the results are directly applicable to adsorption at the air-water interface, with the oil phase treated as air. The same analysis is valid for the attachment of a spherical composite colloidosome capsule pre-infused with the same fluid phase as the supra-particle. As shown previously by many authors (see e.g. ref. 7), the attachment energy of a single 

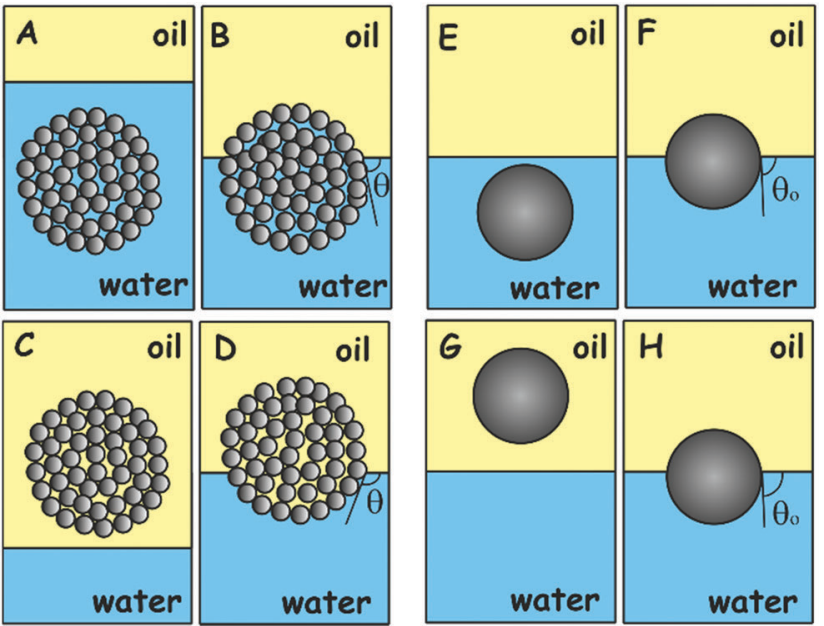

Scheme 1 Schematic representation of the process of attachment of a spherical composite supra-particle built of smaller colloid particles to a liquid-fluid interface: attachment of a water-infused supra-particle from the aqueous phase (A) to the oil-water interface (B); attachment of an oil-infused supra-particle from the oil phase (C) to the oil-water interface (D). Note that the contact angle of the water-infused supra-particle adsorbed at the oil-water interface (A) and (B) is different from the contact angle of the same supra-particle infused initially with oil (C) and (D). Our theoretical model assumes that the oil-water interface is attached only on the surface layer of colloid particles at the supra-particle. (E and F) Represent the attachment of a single smooth solid particle from the water phase (E) to the oil-water interface (F). (G and $H)$ Represent the process of attachment of a single smooth solid particle from the oil phase (G) to the oil-water interface $(\mathrm{H})$. Note that the contact angle of the smooth solid particle at the oil-water interface is the same regardless of the fluid phase the particle comes from, i.e. (F) is to the same as $(H)$. For the case of particle attachment to an air-water interface, the same scheme is valid with the oil phase being replaced by air in $(A)-(H)$.

non-porous colloid particle of radius $a$ and contact angle $\theta_{0}$ to the oil-water interface is:

$$
\begin{gathered}
\Delta E_{1}=-\pi a^{2} \gamma\left(1-\cos \theta_{0}\right)^{2} \quad \text { (from the water phase) } \\
\Delta E_{2}=-\pi a^{2} \gamma\left(1+\cos \theta_{0}\right)^{2} \quad \text { (from the oil phase) }
\end{gathered}
$$

Here, $\gamma$ is the oil-water interfacial tension while $\Delta E_{1}$ and $\Delta E_{2}$ correspond to attachment from the water phase and the oil phase, respectively. Note that according to eqn (1) and (2), the free energy of particle attachment is negative, i.e. the process of particle attachment to the liquid interface is spontaneous from both fluid phases, oil or water. Eqn (1) and (2) also suggest that more energy is required for the removal of a hydrophilic particle $\left(\theta<90^{\circ}\right)$ from the interface into oil than into water, while the opposite is true for a hydrophobic particle $\left(\theta>90^{\circ}\right)$. Similar equations, as (1) and (2), apply for attachment of the particle at the air-water interface with $\gamma$ being the air-water surface tension.

\section{Attachment of a porous supra-particle from the aqueous phase to the oil-water interface}

For the sake of simplicity of analysis here and hereafter we have assumed that the liquid-fluid interface is flat down to the supra-particle three-phase contact line. The process of attachment of the supra-particle (or colloidosome) from the bulk of the aqueous phase to the oil-water interface can be formally considered as a two-step process:

(i) The porous supra-particle displaces a portion of the oil-water interface of area encircled by its contact line:

$$
A_{\mathrm{c}}=\pi r_{\mathrm{c}}^{2}=\pi R^{2} \sin ^{2} \theta
$$

but creates a curved oil-water interface with adsorbed particles of total area $A_{1}$ and a radius of curvature, $R$, roughly the same as that of the adsorbed supra-particle:

$$
A_{1}=2 \pi R^{2}(1-\cos \theta) .
$$

(ii) Only the surface layer of colloid particles within that area, $A_{1}$, on the supra-particle (or colloidosome) surface attach to the oil-water interface. There is experimental evidence for this supra-particle attachment mechanism supported by MRI studies. $^{37}$ For simplicity, the colloidal particles are assumed to be closely-packed together within the porous supra-particle. The possible effects of the degree of fusion of small particles within the supra-particle will not be accounted for as this complicates unnecessarily the analysis, although this can also be incorporated into a more refined model. We will assume that the radius $R$ of the composite supra-particle, is much larger than the radius $a$ of the small particles (building blocks), i.e. $a / R \ll 1$. We will neglect the effect of the curvature of the supraparticle surface on the packing of the small particles and use the well know packing area fractions of spheres in 2D plane. Taking the curvature effects in all these cases gives terms of higher order of magnitude (proportional to the ratio $a / R$ ), which is the reason why we neglect this higher level of detail in our model. The number of attached small particles $N_{1}$ can be calculated by dividing the attached area of the porous supraparticle by the average area per small colloid particle, $A_{\mathrm{s}}$, on its surface.

$$
N_{1}=\frac{A_{1}}{A_{\mathrm{S}}}=2 \eta_{\mathrm{S}} \frac{R^{2}}{a^{2}}(1-\cos \theta)
$$

Here, $\eta_{\mathrm{s}}=\pi a^{2} / A_{\mathrm{s}}$ is the $2 \mathrm{D}$ packing fraction of the small particles on the supra-particle surface. We will consider several possible close packing arrangements of the small colloid particles on the surface of the supra-particle: hexagonal packing, $A_{\mathrm{S}}=2 \sqrt{3} a^{2}$, square packing, $A_{\mathrm{S}}=4 a^{2}$, and $2 \mathrm{D}$ random packing, ${ }^{28} A_{\mathrm{S}}=1.22 \pi a^{2}$, which gives:

$$
\eta_{\mathrm{S}}= \begin{cases}\pi /(2 \sqrt{3}) & \text { (hexagonal packing) } \\ 0.82 & \text { (random packing) } \\ \pi / 4 & \text { (square packing) }\end{cases}
$$

Various experiments with supra-particles made of small latex particles show that the particles arrangement on the supra-particle surface is far from random packing. ${ }^{26,37,42}$ The surface consists primarily of hexagonally packed small particle domains and small areas of square packing domains with defects in between them. Since random close packing area fraction is bracketed in between 
the hexagonal packing $\left(\eta_{\mathrm{S}} \approx 0.907\right)$ and the square packing $\left(\eta_{\mathrm{S}} \approx 0.785\right)$, we will consider these two limiting cases to explore the differences in small particles packing area fraction on the composite supra-particle wettability. We will estimate the free energy of attachment of the supra-particle to the liquid interface for both types of packing conditions to obtain the link between the small particles contact angle and the macroscopic contact angle of the supra-particle at the liquid interface. Thus, the free energy of attachment $\Delta F_{1}$ of the supra-particle (or colloidosome) from the water phase to the oil-water interface can be estimated as follows:

$$
\Delta F_{1}=\gamma A_{1}-\gamma A_{\mathrm{c}}+N_{1} \Delta E_{1} .
$$

The substitution of eqn (1), (3), (4) and (5) into eqn (7) gives:

$$
\Delta F_{1}=-\pi \gamma R^{2}\left\{1-\cos ^{2} \theta+2(1-\cos \theta)\left[\eta_{\mathrm{S}}\left(1-\cos \theta_{0}\right)^{2}-1\right]\right\}
$$

The substitution of eqn (6), into eqn (8) gives:

$$
\Delta F_{1}=-\pi \gamma R^{2}\left\{1-\cos ^{2} \theta+(1-\cos \theta)\left[\frac{\pi}{\sqrt{3}}\left(1-\cos \theta_{0}\right)^{2}-2\right]\right\}
$$

for hexagonal packing and

$$
\Delta F_{1}=-\pi \gamma R^{2}\left\{1-\cos ^{2} \theta+(1-\cos \theta)\left[\frac{\pi}{2}\left(1-\cos \theta_{0}\right)^{2}-2\right]\right\}
$$

for square packing. With respect to the porous supra-particle contact angle, $\theta$, the condition for a minimum of the surface free energy in the system requires that:

$$
\frac{\mathrm{d} \Delta F_{1}}{\mathrm{~d} \cos \theta}=2 \pi \gamma R^{2}\left\{\cos \theta+\left[\eta_{\mathrm{S}}\left(1-\cos \theta_{0}\right)^{2}-1\right]\right\}=0
$$

Depending on the arrangement of particles at the supra-particle surface, eqn (10) allow to connect the contact angle of a supraparticle (or colloidosome) $\theta$ with the contact angle $\theta_{0}$ of its building blocks (i.e. the colloid particles on the surface of the porous supra-particle or composite colloidosome membrane, respectively).

$$
\cos \theta=1-\eta_{\mathrm{S}}\left(1-\cos \theta_{0}\right)^{2}
$$

For building block particles arranged in hexagonal packing on the supra-particle surface, eqn (10) gives:

$$
\cos \theta=1-\frac{\pi}{2 \sqrt{3}}\left(1-\cos \theta_{0}\right)^{2} \text {. }
$$

For building block particles arranged in square packing on the supra-particle surface, eqn (11) gives:

$$
\cos \theta=1-\frac{\pi}{4}\left(1-\cos \theta_{0}\right)^{2} .
$$

Note that eqn (11)-(13) do not depend (in first approximation) on the radius of the small colloid particles, $a$ and the radius $R$ of the porous supra-particle. These equations provide the link between the contact angle $\theta_{0}$ of the building block colloid particles and the effective (macroscopic) contact angle $\theta$ of the porous supra-particle (or colloidosome) attached at the liquid interface. Eqn (11) suggests that at $\eta_{\mathrm{S}} \geq 0.5$ there is an upper critical value, $\theta_{0}^{\mathrm{u}}=\arccos \left(1-\sqrt{2 / \eta_{\mathrm{S}}}\right)$, for the contact angle of small particles, above which eqn (11) does not have a solution for the supra-particle contact angle, $\theta$.

The critical contact angle value for hexagonal packing is $\theta_{0}^{\mathrm{u}} \approx 119^{\circ}$ above which eqn (12) does not have a solution for the supra-particle contact angle, $\theta$. If the small particles are arranged in square packing on the supra-particle surface, eqn (13) has no solution for contact angles above $\theta_{0}^{\mathrm{u}} \approx 126^{\circ}$. The interpretation of this fact is that if the particles in the composite porous particle (or colloidosome membrane) are so hydrophobic that their contact angle is larger than these critical values, the water-infused composite porous particle (or colloidosome) would transfer completely from the water phase into the oil rather than attaching at the oil-water interface with a contact angle $\theta$.

Eqn (11)-(13) are original results derived by us in the present work. The substitution of eqn (11) back into eqn (8) gives the free energy of attachment of the water-infused supra-particle (or colloidosome) as a function of its radius $R$ and the contact angle $\theta_{0}$ of the colloid particles on its surface

$$
\Delta F_{1}=-\pi \gamma \eta_{\mathrm{S}}^{2} R^{2}\left(1-\cos \theta_{0}\right)^{4}
$$

Using eqn (6) and (14) one obtains

$$
\begin{gathered}
\Delta F_{1}=-\frac{1}{12} \pi^{3} \gamma R^{2}\left(1-\cos \theta_{0}\right)^{4} \text { (hexagonal packing) } \\
\Delta F_{1}=-\frac{1}{16} \pi^{3} \gamma R^{2}\left(1-\cos \theta_{0}\right)^{4} \quad \text { (square packing) }
\end{gathered}
$$

Eqn (14) and (15) are only valid for $0<\theta_{0}<\theta_{0}^{\mathrm{u}}$.

\section{Attachment of a porous supra-particle from the oil phase to the oil-water interface}

Similar to the considerations in the previous section, the process of supra-particle (or colloidosome) attachment from the oil phase to the oil-water interface (Scheme 1C and D) can also be formally split into two steps:

(i) The porous supra-particle displaces a portion of the oil-water interface of area $A_{c}$, thereby creating a curved oil-water interface of area $A_{2}$ :

$$
A_{2}=2 \pi R^{2}(1+\cos \theta)
$$

(ii) The colloidal particles within the area $A_{2}$ on the supraparticle surface attach to the oil-water interface. The number of adsorbed small colloid particles at the liquid interface is:

$$
N_{2}=\frac{A_{2}}{A_{\mathrm{S}}}=2 \eta_{\mathrm{S}} \frac{R^{2}}{a^{2}}(1+\cos \theta),
$$

where $\eta_{\mathrm{S}}$ is the packing area fraction of small particles (building blocks) on the surface of the supra-particle, given by eqn (6). Thus, the free energy of attachment of the porous supraparticle (or colloidosome) from the oil phase to the oil-water interface is:

$$
\Delta F_{2}=\gamma A_{2}-\gamma A_{\mathrm{c}}+N_{2} \Delta E_{2}
$$


The substitution of eqn (2), (3), (16) and (17) into eqn (18) gives the following expression for the free energy of supra-particle attachment:

$$
\Delta F_{2}=-\pi \gamma R^{2}\left\{1-\cos ^{2} \theta+2(1+\cos \theta)\left[\eta_{\mathrm{S}}\left(1+\cos \theta_{0}\right)^{2}-1\right]\right\}
$$

Combining eqn (6) and (19) we obtain:

$$
\Delta F_{2}=-\pi \gamma R^{2}\left\{1-\cos ^{2} \theta+(1+\cos \theta)\left[\frac{\pi}{\sqrt{3}}\left(1+\cos \theta_{0}\right)^{2}-2\right]\right\}
$$

for hexagonal packing and

$$
\Delta F_{2}=-\pi \gamma R^{2}\left\{1-\cos ^{2} \theta+(1+\cos \theta)\left[\frac{\pi}{2}\left(1+\cos \theta_{0}\right)^{2}-2\right]\right\}
$$

for square packing of the small particles on the surface of the supra-particle. The condition for the minimum of the surface free energy in the system with respect to the supra-particle macroscopic contact angle requires that

$$
\frac{\mathrm{d} \Delta F_{2}}{\mathrm{~d} \cos \theta}=2 \pi \gamma R^{2}\left\{\cos \theta+\left[1-\eta_{\mathrm{S}}\left(1+\cos \theta_{0}\right)^{2}\right]\right\}=0 .
$$

Eqn (22) is analogous to eqn (10) and provides the relationship between the porous supra-particle (or colloidosome) contact angle $(\theta)$ and small particles' contact angle $\left(\theta_{0}\right)$ upon attachment from the oil phase to the oil-water interface

$$
\cos \theta=\eta_{\mathrm{S}}\left(1+\cos \theta_{0}\right)^{2}-1 \text {. }
$$

Using eqn (6) one obtains

$$
\cos \theta=\frac{\pi}{2 \sqrt{3}}\left(1+\cos \theta_{0}\right)^{2}-1 \quad \text { (for hexagonal packing) }
$$

and

$$
\cos \theta=\frac{\pi}{4}\left(1+\cos \theta_{0}\right)^{2}-1 \quad \text { (for square packing). }
$$

One should note that the values of the supra-particle (or colloidosome) equilibrium contact angle at the oil-water interface predicted by eqn (23)-(25) differ from those predicted by eqn (11)-(13). There is no contradiction here. This is due to the fact that the porous supra-particle (or colloidosome) is filled with water in the case of eqn (11)-(13) and with oil in the case of eqn (23)-(25). This is a fundamental result, which differentiates the attachment of composite porous supra-particles or colloidosome capsules from the attachment of the building block solid colloid particles. The latter would acquire the same equilibrium contact angle $\theta_{0}$ when attached from either the water or oil phase to the oil-water interface. Eqn (23) suggests that at $\eta_{\mathrm{s}} \geq 0.5$ there is a lower critical value for the contact angle of small particles $\theta_{0}^{1}=\arccos \left(\sqrt{2 / \eta_{\mathrm{S}}}-1\right)$ below which eqn (23) does not have a solution for the supra-particle contact angle, $\theta$. The lower critical values are $\theta_{0}^{1} \approx 61^{\circ}$ and $\theta_{0}^{1} \approx 54^{\circ}$ for hexagonal and square packing of spherical colloid particles on the supra-particle (or colloidosome) surface, respectively. This means that, if the small particles in the supra-particle are so hydrophilic that their contact angle is smaller than $\sim 61^{\circ}$, the porous supra-particle pre-filled with oil would transfer completely from the oil phase into the water phase. For the case of a colloidosome initially filled with the oil phase, this model predicts that for hexagonal packing of the small particles of contact angle $\theta_{0}$ smaller than $61^{\circ}$ on the colloidosome membrane, this would produce an oil-in-water Pickering drop rather than an oil-filled colloidosome attached to the oil-water interface with a finite three-phase contact angle, $\theta$.

Similarly to eqn (14) and (15), expressions can be derived for the free energy of attachment of a supra-particle (or colloidosome) from the oil phase to the oil-water interface. The substitution of eqn (23) back into eqn (19) gives the free energy of attachment of an oil-infused porous supra-particle (or colloidosome) as a function of its radius $R$ and the contact angle $\theta_{0}$ of the colloid particles forming the porous supra-particle.

$$
\Delta F_{2}=-\pi \gamma \eta_{\mathrm{s}}^{2} R^{2}\left(1+\cos \theta_{0}\right)^{4}
$$

Using eqn (6) and (26) one derives

$$
\begin{gathered}
\Delta F_{2}=-\frac{1}{12} \pi^{3} \gamma R^{2}\left(1+\cos \theta_{0}\right)^{4} \quad \text { (for hexagonal packing) } \\
\Delta F_{2}=-\frac{1}{16} \pi^{3} \gamma R^{2}\left(1+\cos \theta_{0}\right)^{4} \quad \text { (for square packing) }
\end{gathered}
$$

Eqn (26)-(28) are only valid for $\theta_{0}^{1} \leq \theta_{0} \leq \pi$. Once again, the attachment energy of the porous supra-particle (or colloidosome) from the bulk oil to the oil-water interface is independent, in the first approximation, of the size of the colloid particles on their surface. This result holds for both supra-particles and colloidosomes with densely packed surfaces with smaller particles. Both eqn (14) and (26) predict that the free energy of attachment is negative and the attachment process is thermodynamically spontaneous irrespective from which fluid phase the supraparticle is coming to attach to the oil-water interface. The energy of attachment is different depending on whether the supra-particle is infused with oil (from the oil phase) or water (from the aqueous phase). Note, however, that the value of the contact angle $\theta_{0}$ corresponds to spontaneous adsorption only for finite values of the particle contact angle, $\theta$, according to eqn (24) and (25) for oil-infused particles. Comparing the attachment energy of the porous spherical composite supraparticle, eqn (14) and (26), with that for the attachment of its building blocks (small spherical colloid particles), eqn (1) and (2), one sees a much stronger dependence on the contact angle $\theta_{0}$ of the small particles on the attachment energy of the composite supra-particle.

\section{Materials and methods}

\section{Materials}

Surfactant-free polystyrene (PS) sulphate latex particles with diameter $2.5 \pm 0.3 \mu \mathrm{m}$ were obtained from Invitrogen as an $8.1 \mathrm{wt} \%$ aqueous suspension. The average area per sulphate group on the particles surface is $3.84 \mathrm{~nm}^{2}$, and the number of charged groups per particle is approximately $6 \times 10^{6}$ according to the manufacturer specification. Before use, the sulphate 
latex particles were washed two times with deionised water to remove any impurities. Hexadecane (Reagent Plus 99\%, Sigma) was purified by passing it three times through activated aluminium oxide (STD Grade, Merck) to remove any polar impurities from the oil. Gellan gum (Kelcogel ${ }^{\circledR}$ ), was a gift from CPKelco (USA). Sylgard 184 curable elastomer (polydimethylsiloxane, PDMS) was obtained from Dow Corning. Strata C18-silica functionalised chromatographic column (Gigatube, $60 \mathrm{~mL}$, Phenomenex) was used to remove any hydrophobic or surface active impurities from aqueous gellan gum solutions before the GTT experiment. Ethylenediaminetetraacetic acid disodium salt (EDTA, 99.6\%), methanol (99\%), ethanol (99\%) and sodium hydroxide $(\mathrm{NaOH}$, 99.6\%) were purchased from Sigma. Sodium chloride $(\mathrm{NaCl}$, 99.5\%) was supplied by BDH. Dichlorodimethylsilane (DCDMS, 99.5\%, GC) was obtained from Fluka. All aqueous solutions were prepared with de-ionised water obtained from a Millipore Milli-Q Plus water purification system. The water resistivity was 18.2 $\mathrm{M} \Omega \mathrm{cm}^{-1}$ and its surface tension was $72.3 \pm 0.6 \mathrm{mN} \mathrm{m}^{-1}$ at $20{ }^{\circ} \mathrm{C}$.

\section{Methods}

Preparation of composite porous supra-particles. A glass beaker was pre-cleaned with concentrated solution of $\mathrm{KOH}$ in ethanol for 1 hour, then washed with water and acetone in an ultrasonic bath for $10 \mathrm{~min}$ at room temperature and dried in an oven. The glass beaker was hydrophobised with DCDMS vapours in a sealed box overnight and finally washed with hexane. A $5 \mathrm{wt} \%$ suspension of hydrophobic fumed silica particles (AEROSIL $^{\circledR}$ R202, Degussa) in ethanol was spread uniformly onto the glass beaker inner surface and dried for $20 \mathrm{~min}$ in an oven at $50{ }^{\circ} \mathrm{C}$, thus turning the beaker surface into a superhydrophobic surface. The composite porous particles were prepared using $2.5 \mu \mathrm{m}$ polystyrene sulphate latex particle suspensions of particle concentration of $40 \mathrm{wt} \%$ prepared by settling the original latex suspension at $4000 \mathrm{rpm}$ for $3 \mathrm{~min}$, removing a certain amount of the supernatant and re-dispersing the particles. The concentrated suspension was degassed using a vacuum desiccator. Typically, $10-20 \mu \mathrm{L}$ of $40 \mathrm{wt} \%$ latex particle suspension were placed in a pre-heated glass beaker with superhydrophobic surface and rolled manually by moving the beaker on top of a hot plate set at $90{ }^{\circ} \mathrm{C}$ until the water in the latex suspension droplet evaporated. Finally, the latex particles in the supra-particle aggregate were fused together by thermal annealing in air for 2 hours. This was done by placing the supra-particles inside a dry sample tube immersed in an oil bath heated by a hot plate. The temperature of the bath was maintained close to the glass transition temperature of polystyrene $\left(106-107^{\circ} \mathrm{C}\right)$ by using a temperature probe immersed in the oil and connected to the hot plate control socket. The composite supra-particles obtained had diameters in the range 1.7-2.2 $\mathrm{mm}$ and were stable when immersed in ethanol, hexadecane or water.

Gel trapping technique (GTT) measurements. $2.0 \mathrm{wt} \%$ aqueous solution of gellan was prepared as described in ref. 21-23. Methanolwater mixture ( $50: 50$ by mass) was used as a spreading solvent to deliver the particles both at the air-water and the hexadecanewater interface. The spreading of particles at the air-water surface was achieved by injecting a $10 \mu \mathrm{L}$ aliquot of $0.50 \mathrm{wt} \%$ latex particle dispersion in the spreading solvent at the surface of the hot liquid gellan solution at $55{ }^{\circ} \mathrm{C}$, followed by cooling to $25{ }^{\circ} \mathrm{C}$ to induce gelation. The Petri dish containing this gel layer was sealed in order to prevent evaporation of water from the gel surface. Sylgard 184 curable silicone elastomer (PDMS) mixed with the curing agent (10:1 Sylgard 184 : curing agent) was used to mould the liquid interface after $30 \mathrm{~min}$. For the air-water interface, this was done directly after setting the gel. For the hexadecane-water interface, the hot gellan solution was coated with the pre-warmed hexadecane phase $\left(55^{\circ} \mathrm{C}\right)$. Then, the latex particle spreading and gelling of the aqueous phase were done as for the air-water surface, followed by replacement of the hexadecane phase with curable PDMS. After curing for $48 \mathrm{~h}$ at room temperature, the solid PDMS layer with the trapped latex particles was peeled off the aqueous gel and washed in a hot $10 \mathrm{mM}$ aqueous EDTA solution and hot deionised water at $95{ }^{\circ} \mathrm{C}$ to remove any gel residues from the PDMS surface. The PDMS-particle samples were prepared for SEM imaging by coating them with a carbon nanolayer $(\sim 10 \mathrm{~nm})$ using an Edwards High Vacuum Evaporator.

Sonication method to determine the equilibrium attachment position of fluid-infused porous particles at air-water and oil-water interfaces. Mechanical vibrational method was developed using two different approaches to find the global equilibrium contact angle of the porous particle, i.e. by using an ultrasonic probe or ultrasonic bath. The dry porous supra-particles were placed at the air-water surface in a water-filled cuvette immersed in a beaker with water. A Vibra-Cell ultrasonic probe (3 $\mathrm{mm}$ in diameter, Sonics \& Materials) was immersed inside the water in the beaker and operated in continuous mode at different amplitudes ranging from $0 \%$ to $100 \%$ for up to 10 min (Fig. 1).

In the experiments with pre-wet porous supra-particles, the dry porous particles were initially washed with ethanol to extract all the air from the pores, then immersed in deionised water overnight and finally the water was sucked out almost completely until the particle was covered with a thin layer of water. Then, sonication was applied for different periods of time (1-30 minutes) using an ultrasonic bath (U100, Ultrawave) or an ultrasonic probe (in a separate beaker filled with water) for the attached porous particle to reach global equilibrium state at the liquid interface. Then, water was carefully added in the cuvette to raise the attached particles gradually until they were clearly seen in the field of view of the camera for taking side images. In the experiments at the oil-water interface, the dry supra-particles were first impregnated with ethanol followed by hexadecane. The cuvette was filled with water, hexadecane added on top of it, then the supra-particles were placed at the oil-water interface from the oil side. Different images were taken before and after attachment. Then, sonication was applied for different periods of time (1-20 min) using an ultrasonic bath. This sonication method allowed us to bypass the local energy minima during the particle attachment which would manifest itself in a huge contact angle hysteresis in the absence of vibrations. 


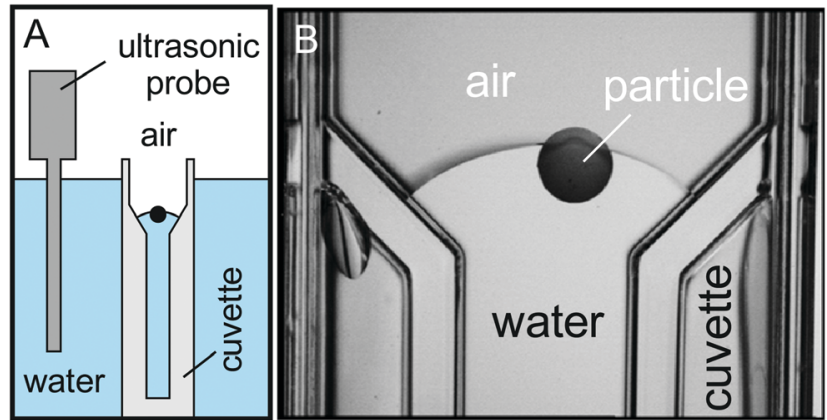

Fig. 1 (A) A diagram of experimental set-up for sonication of dry porous supra-particles at the air-water interface using an ultrasonic probe. (B) A photograph of dry porous supra-particle with diameter $1.7 \pm$ $0.2 \mathrm{~mm}$ attached at the air-water interface before sonication.

\section{Results and discussion}

Contact angle of a porous supra-particle (or colloidosome) attached to the oil-water interface from the aqueous phase

Fig. 2 shows the theoretical dependence of the macroscopic three-phase contact angle, $\theta$, of a water-filled porous supra-particle attached at the oil-water interface as a function of the three-phase contact angle of its "building block" colloid particles, $\theta_{0}$, for hexagonal (solid line) and square (dashed line) packing of the colloid particles on the supra-particle surface. For both packing arrangements, there is an upper critical contact angle of the colloid particles at which the supra-particle contact angle reaches $180^{\circ}$. The critical angle is $\approx 119^{\circ}$ for hexagonal packing and $\approx 126^{\circ}$ for square packing. Beyond these critical values of $\theta_{0}$, eqn (12) and (13) have no real physical solution for $\theta$, i.e. the minimum of the free surface energy of the system corresponds to a complete transfer of the supra-particle into the oil phase $\left(\theta=180^{\circ}\right)$. Note that this analysis also holds for the adsorption of water-filled supra-particles from the water to the air-water interface.

The same analysis is valid for a water-filled colloidosome made from the same building block particles. These equations predict that beyond the critical value of $\theta_{0}$, as pointed out above, a colloidosome would spontaneously transfer through the air-water surface and form a liquid marble,${ }^{32-36}$ however, such high values for the small particle contact angle, $\theta_{0}$, which would allow such a prediction of the theory to be tested experimentally, are difficult to achieve at the air-water interface for smooth surfaces.

Fig. 3A shows the attachment free energy of the supra-particle versus its macroscopic contact angle for hexagonal packing of the building block particles on its surface while Fig. 3B corresponds to square packing. One sees that each free energy curve, which corresponds to a fixed value of building block particles contact angle $\theta_{0}$, has a minimum at different positions for hexagonal and square packing. In this case, the macroscopic contact angle of the supra-particle, $\theta$, which can be directly calculated from eqn (12) or (13) for the respective value of $\theta_{0}$ corresponds to the minimum of the free energy curve presented on the graph.

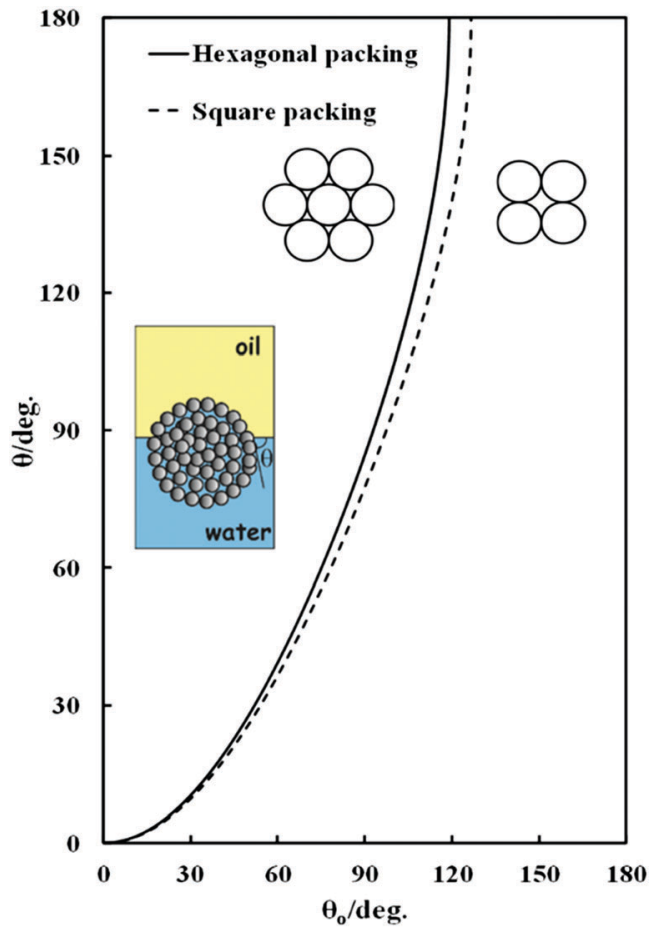

Fig. 2 Theoretical dependence of the macroscopic three-phase contact angle, $\theta$, of a water-filled porous supra-particle (or a colloidosome) attached at the oil-water interface as a function of the three-phase contact angle of their "building block" colloid particles, $\theta_{0}$, for hexagonal or square packing of the building block particles at the supra-particle surface. The inset illustrates the case of water-infused composite supraparticle attached at the oil-water interface.

\section{Contact angle of a porous supra-particle (or colloidosome) attached to the oil-water interface from the oil phase}

Impregnation of the pores in the porous supra-particles with oil or keeping it dry, i.e. full of air, has a different effect on its adsorption behaviour compared with water-impregnated porous particles made of the same building block particles. This affects the attachment energy of the supra-particles at the liquid interface and their equilibrium macroscopic contact angle, $\theta$, at the liquid surface. One should note that eqn (11)-(13) and (23)-(25) predict different values of the porous supra-particles equilibrium contact angle at the oil-water interface, depending on the fluid phase of origin. These results are not contradictory. They are due to the fact that the porous supra-particle is filled with water in the case of eqn (11)-(13), but with oil in the case of eqn (23)-(25). According to eqn (24) for hexagonal packing of the small particles on the supra-particle surface, there is a lower critical value of the colloid particle contact angle: $\theta_{0}^{1} \approx 61^{\circ}$. For square packing conditions, eqn (25) yields a critical value of $\theta_{0}^{1} \approx 54^{\circ}$. Below those critical values eqn (24) and (25) do not have a real physical solution for the porous supra-particle contact angle, $\theta$. This means that, if the small particles in the porous supra-particles' surface are so hydrophilic that their contact angle is smaller than $\sim 61^{\circ}$ or $\sim 54^{\circ}$, the porous supra-particle pre-filled with oil would transfer completely from the oil phase into the water. 

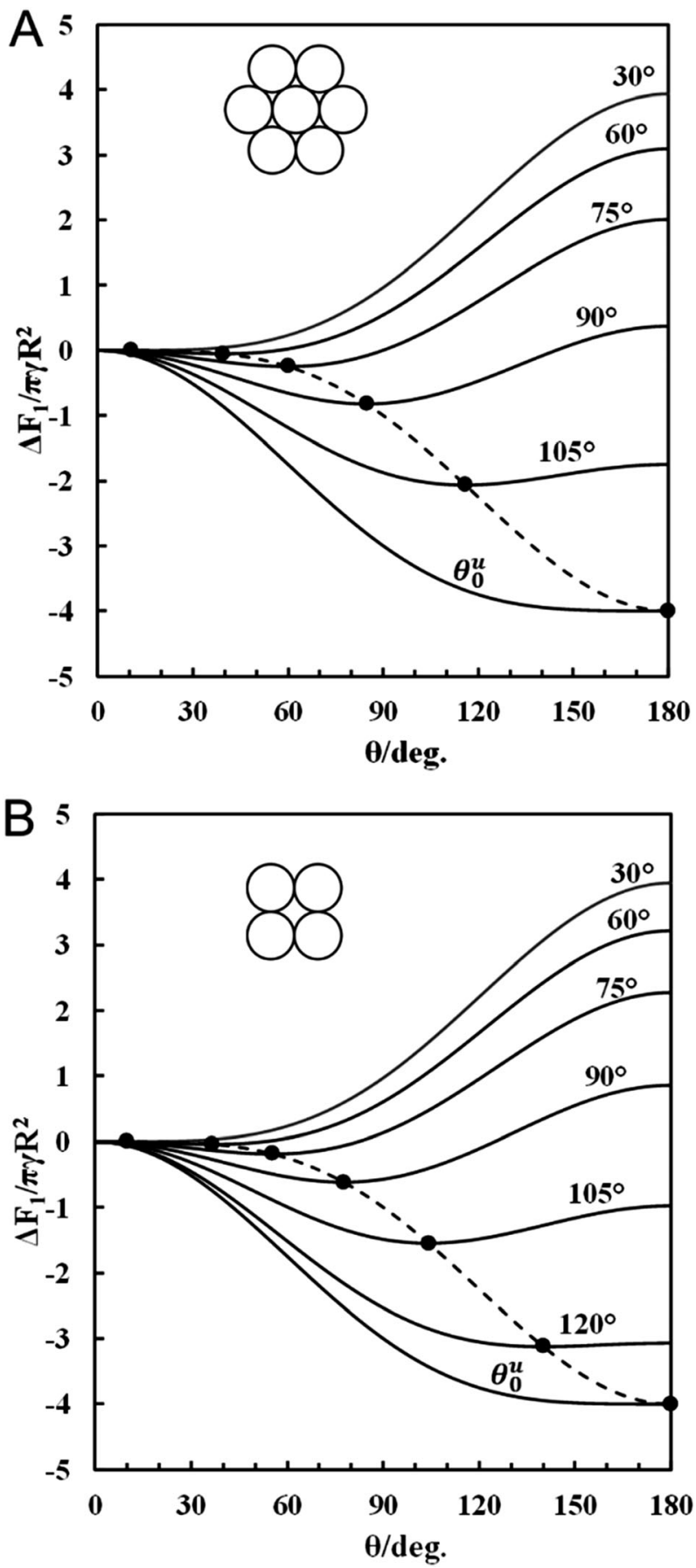

Fig. 3 Dimensionless free energy of attachment for a supra-particle impregnated by water from the water phase to the oil-water interface as a function of its macroscopic contact angle, $\theta$. The colloid particles surface density on the supra-particle surface correspond to (A) hexagonal packing (see eqn (9a)) and (B) square packing (see eqn (9b)). The solid lines correspond to selected values of the contact angle of building block colloid particles, $\theta_{0}$; the lowest curves correspond to the critical $\theta_{0}$ values, $\theta_{0}^{u}$, approx. equal to $119^{\circ}(A)$ and $126^{\circ}$ (B). The filled circles ( ) represent the global minimum of the adsorption free surface energy of the porous supra-particle, with respect to its contact angle for a fixed value of the contact angle of the building block particles, $\theta_{0}$. In this case, the macroscopic contact angle of the supra-particle, $\theta$, is determined from eqn (12) or (13), for the respective value of $\theta_{0}$, which corresponds to the minimum of the free energy curves presented on these graphs. The dashed lines correspond to the minimum free energy of attachment for a supra-particle calculated by eqn (15a and b).
Similarly to eqn (14), the energy of adsorption of the supraparticle from the oil phase to the oil-water interface is given by eqn (26). The free energy of attachment of the porous supraparticle at the oil-water interface is independent (in first approximation) of the size of the colloid particles on its surface and has negative values i.e. corresponds to spontaneous attachment for finite values of the particle contact angle greater than its lower critical value, $\theta_{0}^{1}=\arccos \left(\sqrt{2 / \eta_{\mathrm{S}}}-1\right)$. The minimum of the surface free energy depends on the individual building block contact angle. For example, if the building block particle's contact angle is $\theta_{0}=119^{\circ}$, then the porous supra-particle contact angle would be $139^{\circ}$ and $142^{\circ}$ for square and hexagonal packing, respectively. However, if the individual particles are hydrophilic (i.e. $\theta_{0} \approx 61^{\circ}$ ), then the porous supra-particles' contact angle could be $\theta \approx 43^{\circ}$ for square packing and $\theta_{0} \approx 0^{\circ}$ for hexagonal packing conditions.

Fig. 4 illustrates the theoretical dependence of the threephase contact angle, $\theta$, of an oil-filled porous supra-particle adsorbed at the oil-water interface and the three-phase contact angle of their "building block" colloid particles, $\theta_{0}$. The solid line represents the macroscopic contact angle for the "building block" colloid particles arranged in a hexagonal packing at the surface of the porous supra-particles (i.e. $\theta$ reaches zero at approximately $\theta_{0} \approx 61^{\circ}$ ). On the other hand, for square packing of the colloid particles on the supra-particle surface (the dashed line), $\theta$ reaches zero at a critical contact angle value of $\theta_{0} \approx 54^{\circ}$. Below these critical values of $\theta_{0}$, free energy of attachment of the supra-particle to the oil-water interface has no minimum as a function of $\theta$. Note that this analysis also holds for the adsorption of a dry, air-filled supra-particle from the air phase to the air-water interface. The same analysis would be valid for a hollow, air-filled colloidosome capsule made from the same building block particles.

Fig. 5 gives the surface free energy of attachment at the oil-water interface of an oil-infused porous supra-particle versus the macroscopic supra-particles contact angle, $\theta$, for several fixed values of the contact angle of building block particles, $\theta_{0}$. The filled circle on each curve represents the minimum of the free energy of the system. In this case, the macroscopic contact angle of the supra-particle, $\theta$, can be determined from eqn (24) and (25) for the respective value of $\theta_{0}$, which corresponds to the minimum of the free energy curve presented on this graph.

Depending on building block particles' contact angle, $\theta_{0}$, Fig. 5A and $\mathrm{B}$ represent the free energy required by porous supra-particles to attach to the liquid-fluid interface. For instance, if the building block is hydrophilic $\left(\right.$ e.g. $\left.\theta_{0}=75^{\circ}\right)$, then the predicted contact angle for porous supra-particles would be $\theta \approx 64^{\circ}$ for hexagonal packing or $\theta \approx 76^{\circ}$ for square packing. In comparison, with hydrophobic building block particles (e.g. $\left.\theta_{0} \approx 105^{\circ}\right)$, the predicted supra-particle contact angles would be $\theta \approx 125^{\circ}$ and $\theta \approx 120^{\circ}$ for square and hexagonal packing, respectively. One can also see that the attachment energy of the supra-particle at the oil-water interface does not depend on the size, $a$, of its building blocks particles but depends on the square of the supra-particle radius, $R$. The attachment energy is always negative and corresponds to spontaneous 


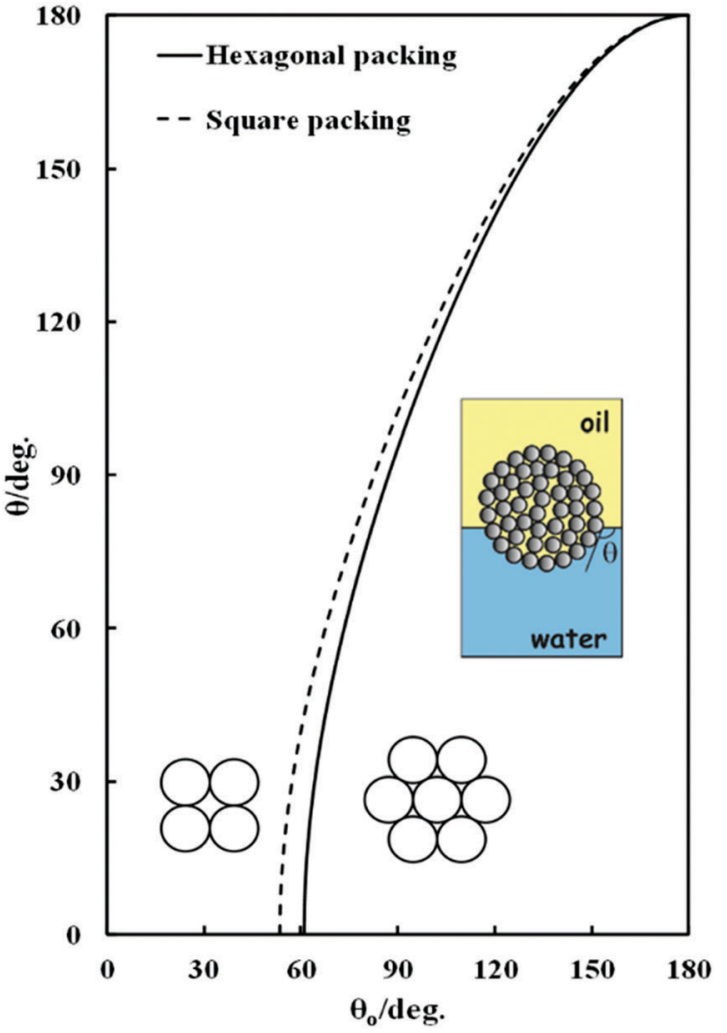

Fig. 4 Theoretical dependence of the macroscopic three-phase contact angle, $\theta$, of an oil-filled porous supra-particle (or a colloidosome) adsorbed at the oil-water interface as a function of the three-phase contact angle of its "building block" colloid particles, $\theta_{0}$, for hexagonal or square packing of the building block particles at the supra-particle surface.

attachment for finite values of the particle contact angle $\theta_{0}$, higher than the lower critical value of $\theta_{0}^{1} \approx 61^{\circ}$ (for hexagonal packing) or $\theta_{0}^{1} \approx 54^{\circ}$ (for square packing). Note that according to eqn (27) and (28), the attachment energy of a hexagonally packed supra-particle is greater in magnitude than that of square-packed supra-particles. This result is not trivial as macroscopic contact angles of the supra-particles are also different for hexagonal and square packing on the particle surface.

Contact angle of sulphate latex microparticles at the air-water interface studied using the gel-trapping technique

The gel-trapping technique was used to measure the three-phase contact angle of $2.5 \mu \mathrm{m}$ sulphate latex particles (used as building blocks in the supra-particles) at the air-water $(\mathrm{a} / \mathrm{w})$ and the hexadecane-water $(\mathrm{o} / \mathrm{w})$ interfaces. Fig. 6 shows the position of the sulphate latex particle on the surface of the PDMS from the GTT experiment after injecting particles' suspensions through the air (A) or water (B) at the air-water interface. It was observed that the wettability of non-porous building block particles does not significantly change, as they are adsorbed from both the aqueous phase and the air phase. For individual sulphate latex particles adsorbed from the nonpolar phase (i.e. air), the contact angle was $63 \pm 3^{\circ}$, and from the polar phase, the contact angle was $65 \pm 1^{\circ}$ at air-water interfaces. A similar observation was obtained when the particles were injected close to the oil-water interface in the
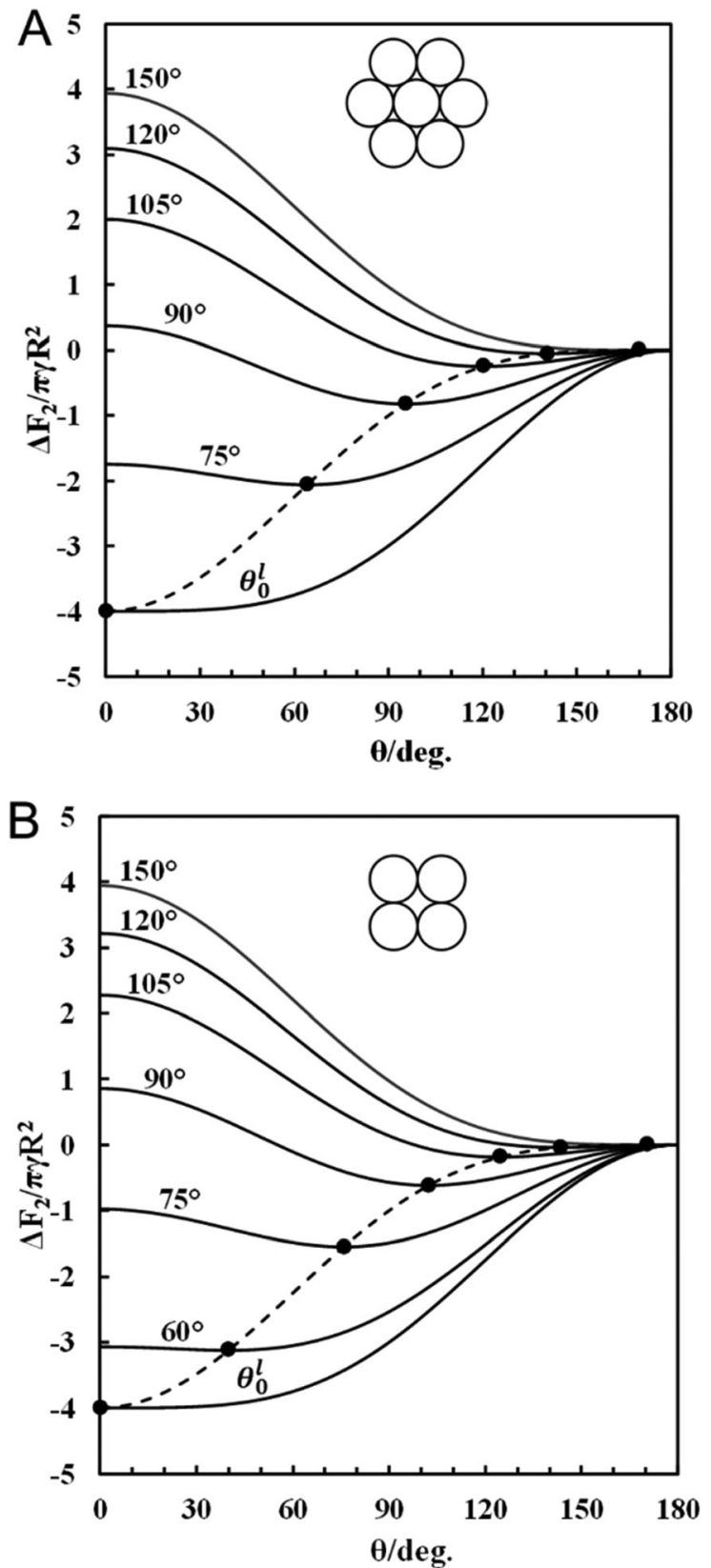

Fig. 5 Dimensionless free energy of attachment for a supra-particle impregnated with oil from the oil phase to the oil-water interface as a function of its macroscopic contact angle, $\theta$. The colloid particles surface density on the supra particle surface correspond to (A) hexagonal packing and (B) square packing (see eqn (20) and (21), respectively). The different lines correspond to selected values of the contact angle of building block colloid particles; the lowest curves correspond to the lower critical $\theta_{0}$ values, $\theta_{0}^{\mathrm{l}} \approx 61^{\circ}$, (A) and $\theta_{0}^{\mathrm{l}} \approx 54^{\circ}$ (B). The filled circles $(\bullet)$ on the curves represent the global minimum of the adsorption free surface energy of the porous supra-particle with respect to its contact angle for a fixed value of the contact angle of the building block particles, $\theta_{0}$. The dashed lines correspond to the minimum free energy of attachment for a supra-particle calculated by eqn (27) and (28).

hexadecane (C) or in the water (D). The results show that the adsorption of particles from hexadecane is practically the same as for the particles adsorbed from the water phase. The contact angle 


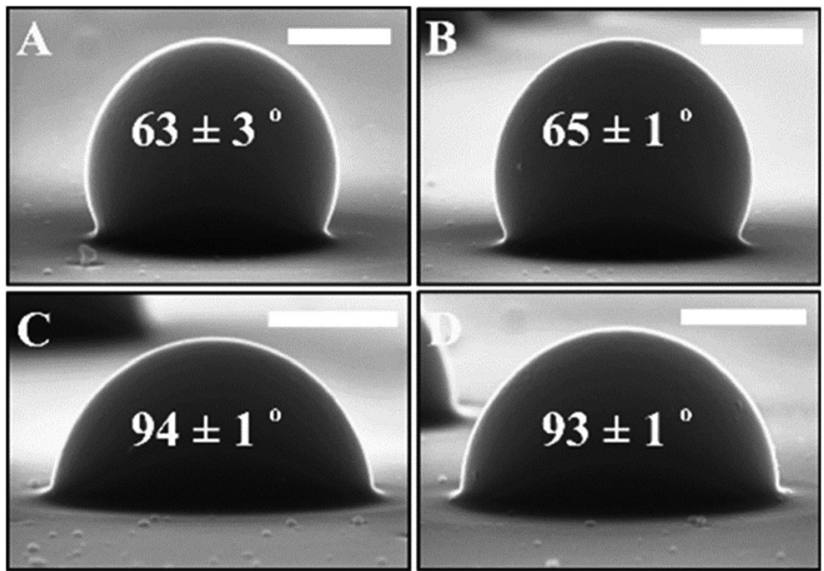

Fig. 6 SEM micrograph of sulphate latex particles of an average diameter of $2.5 \pm 0.3 \mu \mathrm{m}$ imaged with a GTT for determining their three-phase contact angle $\theta_{0}$ at: (A) air-water interface, the particle suspensions dispersed in ethanol were injected from the air phase; (B) air-water interface, the particles were injected from the water phase; (C) hexadecanewater interface, particle suspensions dispersed in ethanol were injected from the hexadecane phase; and (D) hexadecane-water interface, particles were injected from the water phase. The value of $\theta_{0}$ was averaged out of 10 different particles protruding from the PDMS substrate obtained by templating the particles at the liquid interface. The scale bar is $1 \mu \mathrm{m}$ on all images.

observed for the building block latex particles adsorbed from the water phase was $94 \pm 1^{\circ}$, and from the hexadecane phase, it was $93 \pm 1^{\circ}$ at the oil-water interface. These data provide a general idea of the wettability of the building blocks of the composite porous supra-particles, which are made of the same particles.

Using those measured $\theta_{0}$ values, the theory predicts that the contact angle of the porous supra-particles impregnated with water at the air-water interface would be in the range $41^{\circ}-44^{\circ}$ for a square packing and $44^{\circ}-47^{\circ}$ for a hexagonal packing of the latex particles, whereas the porous supra-particles impregnated with water adsorbed to the oil-water interface could be in the range $88^{\circ}-92^{\circ}$ for a hexagonal packing and $81^{\circ}-84^{\circ}$ for a square packing of the latex particles. The theory for porous supraparticles with pores filled with air predicts that the supraparticle contact angle ranges from $0^{\circ}$ to $37^{\circ}$ for building block particles arranged in a hexagonal packing on their surface, whereas for an arrangement of square packing, it ranges from $40^{\circ}$ to $56^{\circ}$. In contrast, for supra-particle pores impregnated with hexadecane regarding its adsorption from the hexadecane phase to the hexadecane-water interface, the theory predicts that the contact angle of the porous supra-particles at an oil-water interface ranges from $101^{\circ}$ to $104^{\circ}$ for a hexagonal packing and from $107^{\circ}$ to $110^{\circ}$ for a square packing. Sonication method for determining the global equilibrium contact angle of porous supra-particles at the air-water and oil-water interfaces.

The aim of this method was to find the equilibrium contact angle of porous particles attached to $\mathrm{a} / \mathrm{w}$ or $\mathrm{o} / \mathrm{w}$ interfaces by using vibrational energy generated from an ultrasonic bath or ultrasonic probe as described earlier. The results are shown for supra-particle contact angle at different amplitudes or times of vibration to reach equilibrium adsorption. Sonication was used to overcome the energy barrier, which exists between the global energy minimum and local energy minima for different attachment positions of the porous particles and the liquid interface. The local minima are a result of pinning of the liquid interface on the small particle building blocks at the supra-particle surface which leads to contact angle hysteresis. By vibrating the system with sonication we facilitate the movement of the supra-particle position towards the global minimum of the free energy, as predicted by the theory. Similar effects appear in practice in many formulation processes during mechanical homogenisation which is used when Pickering emulsions are formed.

\section{Contact angle of dry porous supra-particles sonicated} at the air-water interface

Dry porous supra-particles were placed onto the a/w surface and vibrated in this configuration using an ultrasonic probe in water ( $3 \mathrm{~mm}$ in diameter, Vibra-Cell from Sonics and Materials) at different amplitudes ranging from $0 \%$ to $100 \%$ for 10 minutes. The observed contact angles are summarized in Fig. 7. The dry porous supra-particles tended to be more hydrophilic, as predicted. This method allowed for moving the particle from its initial metastable state, as its adsorption position adjusts towards the equilibrium state because of the vibration. Fig. 8 shows the change of the particles contact angle after sonication at different amplitudes for $10 \mathrm{~min}$, representing the effect of an increase in vibration power. This contact angle is close to the

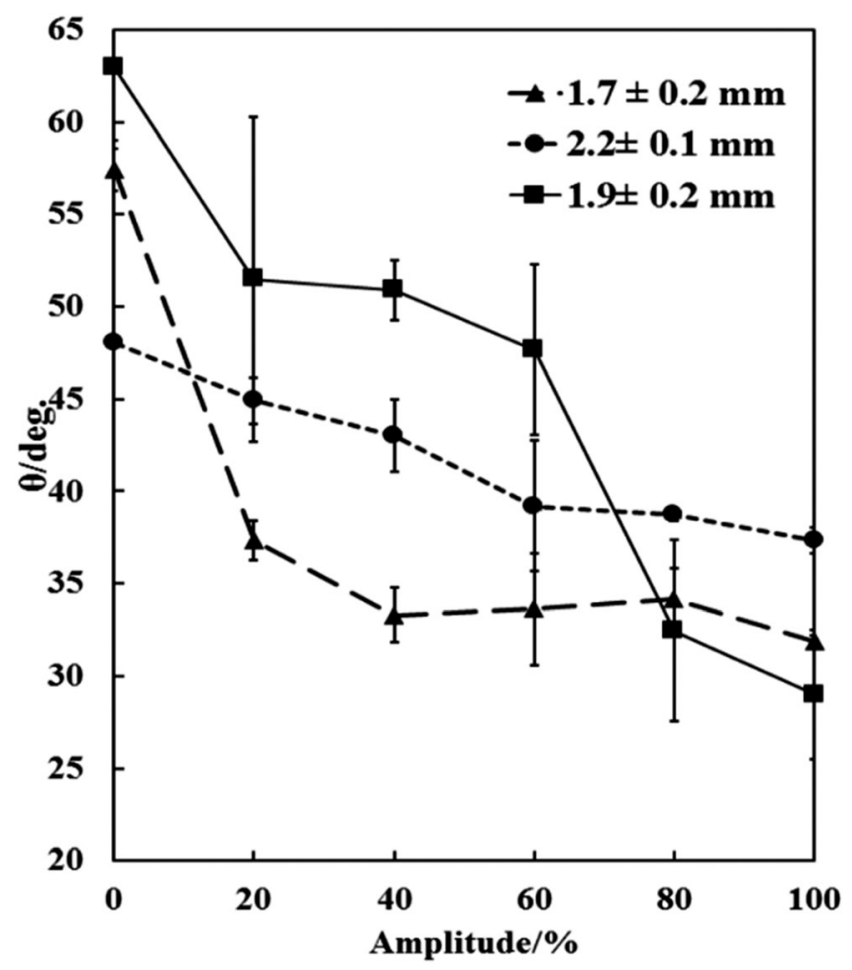

Fig. 7 Contact angle of dry porous particles at the a/w interface after applying ultrasonication at different amplitudes, which reflects the drift of the contact angle from the initial metastable state towards an equilibrium position. The sonication time is fixed to $10 \mathrm{~min}$. The contact angle is reduced from a higher contact angle to the lowest possible after sonicating the system with the attached porous supra-particles. 


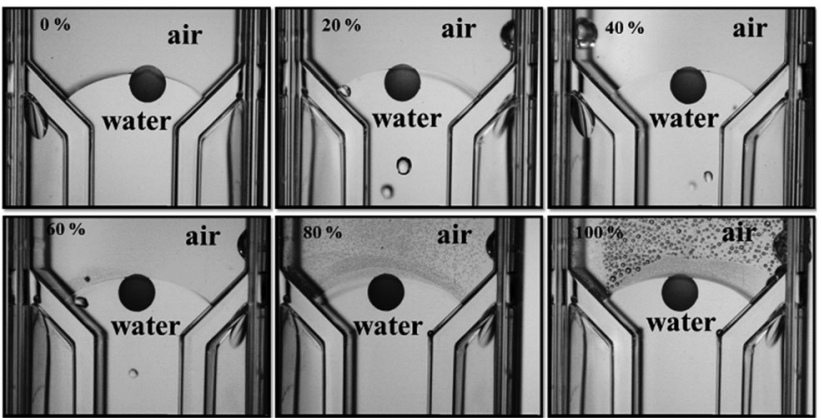

Fig. 8 Side images of initially dry porous supra-particles attached to the air-water interface after ultrasonication at different power amplitudes in a custom designed cuvette. The supra-particle diameter is $1.7 \pm 0.2 \mathrm{~mm}$.

theoretical results, which predict that the contact angle of dry particles at the a/w interface, $\theta_{0}=63^{\circ} \pm 3^{\circ}$, of the small constituting particles. Taking into account the very steep dependence of $\theta$ on $\theta_{0}$ in the vicinity of the critical contact angle of $61^{\circ}$, the theoretical model for the supra-particle adsorption is in a good agreement with the experimental data. The contact angle of the dry porous particles after sonication at different amplitudes represents the change in the contact angle with the increase in sonication power.

The value of the experimentally determined equilibrium contact angle by this method agrees with the theoretical results, which predict that the contact angle of dry supra-particles at the a/w interface for $\theta_{0}=63^{\circ} \pm 3^{\circ}$ of the small "building block" particles will provide an equilibrium contact angle for the porous particles at $\theta=0^{\circ}-37^{\circ}$ in a hexagonal arrangement and $\theta=40^{\circ}-56^{\circ}$ in a square arrangement. Given the accuracy of the GTT measurements for the latex particle contact angle, there is remarkably good agreement between the theory and the experiment (see Fig. 7).

\section{Attachment of water-infused supra-particles sonicated at the air-water interface}

Dry porous particles were initially washed with ethanol to remove all air from the pores. The particles were then incubated in water overnight. After initial attachment at the air-water interface, sonication was employed for different durations (1-30 minutes) using an ultrasonic bath (Ultrawave) or ultrasonic probe (Vibra-Cell). The initial contact angle of the supra-particles sonicated with the ultrasonic probe started at $30^{\circ}$ and ended at $57^{\circ}$, which agreed with the theoretical value of the contact angle of particles (see Fig. 9). The theory predicts that the contact angle of the porous supra-particles adsorbed from water to the a/w interface would be in the range of $41-44^{\circ}$ or $44-47^{\circ}$ for building block particles in square or hexagonal packing, respectively.

\section{Attachment of water-infused porous supra-particles at the hexadecane-water interface}

Water-infused supra-particles were attached to the hexadecanewater interface using a spatula to raise the particle to the liquid interface. The supra-particles were impregnated with Milli-Q water. The cuvette was first filled with water, and then the oil

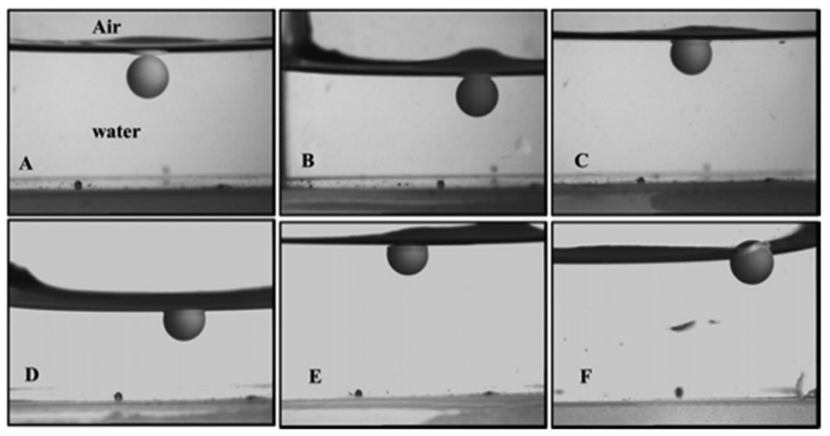

Fig. 9 Side images of water-filled porous supra-particles adsorbed at the a/w interface for different durations of sonication using an ultrasonic bath after: (A) $3 \mathrm{~min}$, (B) $6 \mathrm{~min}$, (C) $9 \mathrm{~min}$, (D) $15 \mathrm{~min}$, (E) $20 \mathrm{~min}$ and (F) $30 \mathrm{~min}$. The supra-particle was attached to the air-water interface after initial sucking and the subsequent insertion of water. The water sucking step corresponds to the a/w surface pressing the particles towards the bottom of the cuvette, while the water addition step lifts the particle and the a/w interface into a viewing position.

phase was added on top of it. Then, the pre-wet particles were attached to the interface through the water phase. Different images were taken before and after sonication. Sonication was applied for different durations (1-20 minutes) using an ultrasonic bath (Ultrawave). A supra-particle of $1.7 \mathrm{~mm}$ in diameter was sonicated at the oil-water interface for differing durations to reach its global equilibrium position and contact angle. The porous water-infused supra-particle increased its contact angle from $12^{\circ}$ (initial attachment) to $77 \pm 4^{\circ}$. In comparison with the theoretical model $\left(81^{\circ}-84^{\circ}\right.$ for square packing and $88^{\circ}-92^{\circ}$ for hexagonal packing), the expected global equilibrium contact angle was slightly higher (see Table 1).

\section{Attachment of porous supra-particles impregnated with hexadecane at the hexadecane-water interface}

The dry supra-particles were first impregnated with the hexadecane phase. After that, the cuvette was filled with water, oil was added on top and then the oil-infused supra-particles were attached to the hexadecane-water interface by dropping them through the hexadecane phase with a spatula. Different images were taken before and after ultrasonication for different durations (1-30 minutes) using an ultrasonic bath (Ultrawave) to reach their equilibrium position at the o/w interface. The initial position of the attached supra-particle corresponded to contact angles between $150^{\circ}$ and $110^{\circ}$, respectively; however, as sonication was applied, the particle contact angle began to gradually decrease and had the potential to change the particle to hydrophilic with a contact angle ranging from $110^{\circ}$ to $71^{\circ}$. The particles that started with a $150^{\circ}$ contact angle remained hydrophobic after sonication with a contact angle of around $110^{\circ}$. In this case, the comparison of the experimental data with the theoretical model also shows that the contact angles observed agree within the range expected by the theory (for square packing, $107^{\circ}-110^{\circ}$, and for hexagonal packing, $101^{\circ}-104^{\circ}$ ), as presented in Table 1 .

These results have potential implication of the pre-infusion of the porous supra-particles (or particle aggregates) with oil or water on their performance as stabilisers of Pickering emulsions. 
Table 1 Summary of the experimental values for porous supra-particles' contact angle impregnated with air, water or hexadecane at the air-water and hexadecane-water interfaces related to the predicted theoretical values. Here $\theta_{0}$ is the contact angle of the small particles (building blocks) while $\theta_{\mathrm{s}}$ and $\theta_{\mathrm{h}}$ are the predicted macroscopic contact angles of the supra-particles assuming close square packing and hexagonal packing of the small particles on their surfaces. $\theta$ is the experimentally determined value of the supra-particle contact angle after initial attachment and after 10 min of sonication, respectively (last two columns of data) at different liquid interfaces and fluids in the pores of the supra-particle. Sonication is applied to overcome the initial pinning of the three-phase contact line

\begin{tabular}{|c|c|c|c|c|c|c|c|}
\hline \multirow[b]{2}{*}{ Liquid interface type } & \multirow[b]{2}{*}{ Fluid inside pores } & \multirow[b]{2}{*}{$\theta_{0} / \mathrm{deg}$} & \multirow[b]{2}{*}{$\theta_{\mathrm{s}} / \mathrm{deg}$} & \multirow[b]{2}{*}{$\theta_{\mathrm{h}} / \mathrm{deg}$} & \multirow{2}{*}{$\begin{array}{l}\text { Porous supra-particle } \\
\text { diameter/mm }\end{array}$} & \multicolumn{2}{|l|}{$\theta / \mathrm{deg}$} \\
\hline & & & & & & Initial & Final \\
\hline $\mathrm{A}-\mathrm{W}$ & Air & $63 \pm 3$ & $40-56$ & $2-37$ & 1.7 & $57 \pm 1$ & $32 \pm 1$ \\
\hline $\mathrm{A}-\mathrm{W}$ & Water & $65 \pm 1$ & $41-44$ & $44-47$ & 1.7 & $28 \pm 2$ & $57 \pm 3$ \\
\hline $\mathrm{O}-\mathrm{W}$ & Hexadecane & $93 \pm 1$ & $81-84$ & $88-92$ & 1.7 & $105 \pm 3$ & $77 \pm 4$ \\
\hline $\mathrm{O}-\mathrm{W}$ & Water & $94 \pm 1$ & $107-110$ & $101-104$ & 1.7 & $12 \pm 3$ & $71 \pm 5$ \\
\hline
\end{tabular}

Our theoretical model predicts different macroscopic contact angles of a supra-particle attached at the oil-water interfaces depending on which liquid (water or oil) has been infused in the pores of the supra-particle before its attachment. This is illustrated in Fig. 10 for the case of hexagonal packing of building block particles at the supra-particle surface. The contact angles, $\theta$, are always different except at two "isogonic" contact angles of the building block particles, $\theta_{0}^{\mathrm{ml}}$ and $\theta_{0}^{\mathrm{mu}}$, where $\theta$ for a water-filled and oil-filled supra particles are the same. Their values can be obtained by equating eqn (11) and (23), which after rearrangements gives

$$
\cos \theta_{0}^{\mathrm{m}}= \pm \sqrt{\frac{1-\eta_{\mathrm{s}}}{\eta_{\mathrm{S}}}}
$$

where $0.5 \leq \eta_{\mathrm{S}} \leq \pi / 2 \sqrt{3}$. These "isogonic" contact angles are $\theta_{0}^{\mathrm{ml}} \approx 71^{\circ}$ and $\theta_{0}^{\mathrm{mu}} \approx 109^{\circ}$ for hexagonal packing, and $\theta_{0}^{\mathrm{ml}} \approx 58^{\circ}$ and $\theta_{0}^{\mathrm{mu}} \approx 122^{\circ}$ for square packing of the small particles at the supra-particle surface. Our model predicts that if the contact angle of the building block particles is smaller than the lower "isogonic" angle (i.e. $\theta_{0}<\theta_{0}^{\mathrm{ml}}$ ), the supra-particle filled with oil should have smaller $\theta$ than that filled with water. In other words, the oil-filled porous supra-particle will be more hydrophilic than the waterfilled supra-particle at the same conditions. Similarly, if the contact angle of the building block particles is bigger than the upper "isogonic" angle $\left(\theta_{0}>\theta_{0}^{\mathrm{mu}}\right)$, the supra-particle filled with water should have bigger $\theta$ than that filled with oil, hence the water-filled porous supra-particle will be more hydrophobic than the oil-filled supra-particle. These results are counterintuitive but are easy to explain with the balance of surface energies of structured supra-particle surface.

However, for the building block particle contact angles $\theta_{0}$ falling within the range between the lower and the upper isogonic contact angle, $\theta_{0}^{\mathrm{ml}}<\theta_{0}<\theta_{0}^{\mathrm{mu}}$, the porous supraparticles infused with oil would be more hydrophobic than the same porous particles infused with water. Since the using moderately hydrophilic particles generally gives oil-in-water Pickering emulsion (e.g. at 50:50 oil-to-water volume ratio), infusing the porous particles with the aqueous phase would make the original particles more hydrophilic. Since using moderately hydrophobic particles as emulsifiers usually gives water-in-oil Pickering emulsions, infusing the porous particles with the oil

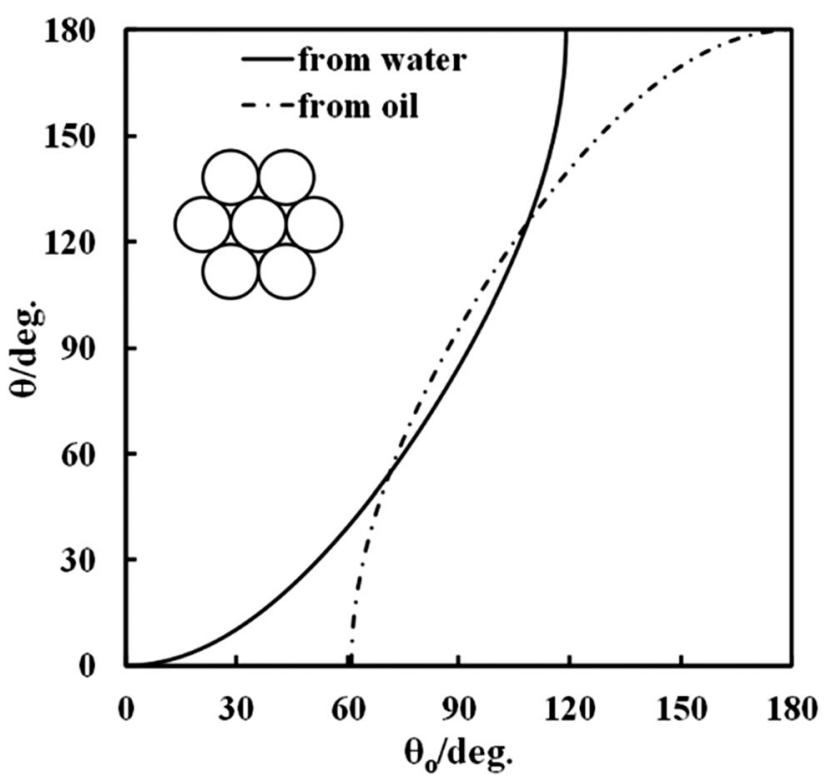

Fig. 10 Theoretical dependence of the macroscopic three-phase contact angle, $\theta$, of a water-filled (solid line) and oil-filled (dash-dot line) porous supra-particle (or a colloidosome) attached at the oil-water interface as a function of the three-phase contact angle of their "building block" colloid particles, $\theta_{0}$, for hexagonal packing of the building block particles at the supra-particle surface.

phase can make them more hydrophobic. Experiments with using aggregated particles as emulsifiers have been reported by different authors ${ }^{29-31}$ and give different results depending on the initial liquid phase with which the particles are mixed introduced in the system. Hence a different type of Pickering emulsion could be obtained at the same other conditions by using the same porous particles infused with the oil phase compared to these infused with the aqueous phase.

Finally, one may speculate what would happen if the building blocks of the supra-particle are not firmly bound to each other and it does not maintain spherical shape upon attaching to the liquid-fluid interface. Most likely, in this case the aggregate would reform its shape from spherical to lens-like geometry upon attachment or partially disintegrate at the liquid interface if the particle adsorption energy is much stronger than the particle-particle interactions within the aggregate. In this 


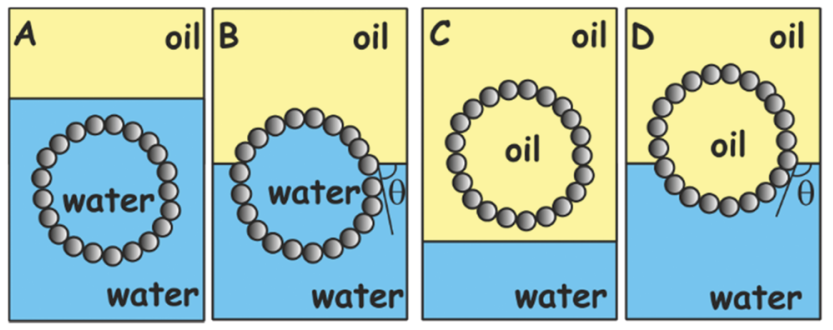

Scheme 2 Schematic representation of the process of attachment of a spherical colloidosome built of smaller colloid particles to a liquid-fluid interface: attachment of a water-infused colloidosome from the aqueous phase (A) to the oil-water interface (B); attachment of an oil-infused colloidosome from the oil phase (C) to the oil-water interface (D). Note that the contact angle of the water-infused colloidosome adsorbed at the oil-water interface (A) and (B) is different from the contact angle of the same colloidosome infused initially with oil (C) and (D). Our theoretical model assumes that the oil-water interface is attached only on the colloidosome membrane built of colloid particles.

extreme case spreading of the softly-bounded particle aggregate at the liquid interface would occur to form a layer of adsorbed particles may occur. This case would require additional information about the particle-particle interactions within the aggregate (supra-particle) and would need a generalisation of our model which works only for spherical composite supraparticles.

\section{Conclusions}

In this study we developed a theoretical model for the attachment of porous supra-particles at a liquid-fluid interface. This model is valid for composite supra-particles built from smaller spherical colloid particles with smooth surfaces, which is usually the case for agglomerated particles in powdered materials. The case of water-infused porous supra-particles attached to the oil-water interface from the water phase was considered as well as the case of oil-infused supra-particles adsorbed at the oil-water interface from the oil-phase. Our model assumes that the particle attachment at the air-water and oil-water interfaces occurs through the adsorption of the surface layer of colloid particles on the composite supra-particle surface. Therefore, the results presented in this study could also be potentially valid for the attachment of fluidinfused colloidosomes to liquid surfaces (see Scheme 2). Different packing conditions of small particles on the supra-particle surface were considered. The connection between the macroscopic contact angle of the porous supra-particles and the contact angle of the small "building block" colloid particles was derived for both types of packing conditions. The model predicts that the supraparticles would have different macroscopic contact angles depending on which fluid phase they originate from before they attach at the oil-water interface. This means that the porous particle would have a different attachment position and macroscopic contact angle if it is infused with water, compared with those of the same particle infused with oil. The macroscopic contact angle of the supra-particles does not depend on the size of its building block colloid particles or the size of the supra-particle itself. This behaviour differs from the attachment of non-porous spherical solid particles of smooth surfaces, which have the same equilibrium contact angle at the liquid interface, independently of the liquid phase from which they originate.

Equations for the energy of attachment of porous spherical supra-particles at the oil-water interface were derived for the cases of initial infusion with water or oil. It was found that for supra-particles infused with water, the supra-particle contact angle at the oil-water interface has a physical solution only for values of the building block particle contact angle smaller than, or equal to, an upper critical contact angle of $\approx 119^{\circ}$ for hexagonal packing or $\approx 126^{\circ}$ for square packing conditions. More hydrophobic small particles would favour a complete transfer of the water-infused supra-particle (or colloidosome) into the oil phase. For supra-particles initially infused with oil, the supra-particle contact angle at the oil-water interface has a physical solution only for values of the building block particle contact angle larger than, or equal to, a lower critical contact angle of $\approx 61^{\circ}$ for hexagonal packing or $\approx 54^{\circ}$ for square packing conditions. More hydrophilic small particle building blocks would favour a complete transfer of the oil-filled supraparticle (or colloidosome) into the water phase.

Our theory predicts that supra-particles with hexagonal packing of small particles would exhibit larger adsorption energy compared with the same size of supra-particle with square packing of building block particles.

To test the theory we produced model porous supra-particles by evaporating latex particle suspensions on super-hydrophobic surface followed by thermal annealing to partially fuse the small latex particle into the structure of the obtained composite supra-particles. The wettability of the model porous supraparticles was studied experimentally at different liquid-fluid interfaces by studying the contact angle for the dry and waterinfused porous supra-particles at a/w interface, and oil-infused or water-infused porous supra-particles at the hexadecanewater interfaces. Since the composite supra-particles remained trapped in a series of metastable states when attached to the liquid surface, they show contact angle hysteresis which is a result of the pinning of the supra-particle contact line on the "building block" latex particles, which prevents spontaneous adjustment towards the equilibrium position. We developed a sonication method which allows the supra-particle position to be adjusted at the liquid interface with its contact angle drifting towards global equilibrium.

The contact angles of the small latex particles were determined experimentally by using the Gel Trapping Technique. Using our theoretical model we calculated the expected supra-particle contact angles at air-water and oil-water interfaces for initial particle infusion with air, water or oil. The latex particle building blocks showed contact angles of about $65^{\circ}$ at the air-water interface and $94^{\circ}$ at the hexadecane-water interface. For dry supraparticles attached to the air-water interface as well as waterinfused supra-particles, a reasonably good quantitative agreement was found between the experimental data and the theoretical predictions for the supra-particle contact angle. The porous 
supra-particles were hydrophilic when pre-filled with water and became hydrophobic when pre-filled with hexadecane.

\section{Acknowledgements}

H. A. thanks the Ministry of Higher Education of the Kingdom of Saudi Arabia for the funding of his PhD studentship. The authors thank Mr Tony Sinclair for the SEM imaging of the GTT sample.

\section{Notes and references}

1 Encyclopaedia of Emulsion Technology, ed. T. F. Tadros and B. Vincent, Marcel Dekker, 1983.

2 J. T. Davies and E. K. Rideal, Interfacial Phenomena, Academic Press, New York, 1963.

3 A. W. Adamson, Physical Chemistry of Surfaces, John Wiley and Sons, New York, 1976.

4 E. M. Furst, Proc. Natl. Acad. Sci. U. S. A., 2011, 108, 20853-20854.

5 W. Ramsden, Proc. R. Soc. London, 1903, 72, 156-164.

6 S. U. Pickering, J. Chem. Soc., Trans., 1907, 91, 2001-2021.

7 V. N. Paunov, B. P. Binks and N. P. Ashby, Langmuir, 2002, 18, 6946-6955.

8 P. A. Kralchevsky, I. B. Ivanov, K. P. Ananthapadmanabhan and A. Lips, Langmuir, 2005, 21, 50-63.

9 E. Dickinson, Curr. Opin. Colloid Interface Sci., 2010, 15, 40-49.

10 E. Dickinson, Adv. Colloid Interface Sci., 2011, 165, 7-13.

11 V. N. Paunov, O. J. Cayre, P. F. Noble, S. D. Stoyanov, K. P. Velikov and M. Golding, J. Colloid Interface Sci., 2007, 312, 381-389.

12 B. S. Murray and R. Ettelaie, Curr. Opin. Colloid Interface Sci., 2004, 9, 314-320.

13 H. A. Wege, S. Kim, V. N. Paunov, Q. Zhong and O. D. Velev, Langmuir, 2008, 24, 9245-9253.

14 Z. P. Du, M. P. Bilbao-Montoya, B. P. Binks, E. Dickinson, R. Ettelaie and B. S. Murray, Langmuir, 2003, 19, 3106-3108.

15 R. G. Alargova, D. S. Warhadpande, V. N. Paunov and O. D. Velev, Langmuir, 2004, 20, 10371-10374.

16 A. L. Campbell, S. D. Stoyanov and V. N. Paunov, Soft Matter, 2009, 5, 1019-1023.

17 R. Aveyard, J. H. Clint and T. S. Horozov, Phys. Chem. Chem. Phys., 2003, 5, 2398-2409.
18 T. S. Horozov, D. A. Braz, P. D. I. Fletcher, B. P. Binks and J. H. Clint, Langmuir, 2007, 24, 1678-1681.

19 N. Ballard and S. A. Bon, J. Colloid Interface Sci., 2015, 448, 533-544.

20 E. L. Sharp, H. Al-Shehri, T. S. Horozov, S. D. Stoyanov and V. N. Paunov, RSC Adv., 2014, 4, 2201-2213.

21 V. N. Paunov, Langmuir, 2003, 19, 7970-7976.

22 O. J. Cayre and V. N. Paunov, Langmuir, 2004, 20, 9594-9599.

23 V. N. Paunov, O. J. Cayre, MRS Proceedings, Fall 2003, M8.25.1-3.

24 L. A. Arnaudov, O. J. Cayre, S. D. Stoyanov, M. Cohen-Stuart and V. N. Paunov, Phys. Chem. Chem. Phys., 2010, 12, 328-331.

25 H. Al-Shehri, T. S. Horozov and V. N. Paunov, Soft Matter, 2014, 10, 6433-6441.

26 V. Rastogi, S. Melle, O. G. Calderon, A. A. Garcia, M. Marquez and O. D. Velev, Adv. Mater., 2008, 20, 4263-4268.

27 S. H. Kim, J. M. Lim, S. K. Lee, C. J. Heo and S. M. Yang, Soft Matter, 2010, 6, 1092-1110.

28 J. G. Berryman, Phys. Rev. A: At., Mol., Opt. Phys., 1983, 27, 1053-1061.

29 B. B. Binks and S. O. Lumsdon, Phys. Chem. Chem. Phys., 2000, 2, 2959-2967.

30 B. P. Binks and J. A. Rodrigues, Langmuir, 2003, 19, 4905-4912.

31 B. P. Binks, J. Philip and J. A. Rodrigues, Langmuir, 2005, 21, 3296-3302.

32 P. Aussillous and D. Quéré, Nature, 2001, 411, 924-927.

33 P. Aussillous and D. Quéré, Proc. R. Soc. A, 2006, 462, 973-999.

34 B. P. Binks and R. Murakami, Nat. Mater., 2006, 5, 865-869.

35 R. Murakami and A. Bismarck, Adv. Funct. Mater., 2010, 20, 732-737.

36 M. Rutkevičius, G. H. Mehl, J. T. Petkov, S. D. Stoyanov and V. N. Paunov, J. Mater. Chem. B, 2015, 3, 82-89.

37 H. Al-Shehri, T. S. Horozov and V. N. Paunov, Soft Matter, 2016, DOI: 10.1039/c6sm01651k.

38 X.-C. Luu, J. Yu and A. Striolo, J. Phys. Chem. B, 2013, 117, 13922-13929.

39 M. Luo, O. A. Mazyar, Q. Zhu, M. W. Vaughn, W. L. Hase and L. L. Dai, Langmuir, 2006, 22, 6385-6390.

40 M. Luo and L. L. Dai, J. Phys.: Condens. Matter, 2007, 19, 375109.

41 D. S. Frost and L. L. Dai, J. Chem. Phys., 2012, 136, 084706.

42 O. D. Velev, A. M. Lenhoff and E. W. Kaler, Science, 2000, 287, 2240-2243. 